\title{
Laser UV Microirradiation of Interphase Nuclei and Post-Treatment with Caffeine
}

\author{
A New Approach to Establish the Arrangement \\ of Interphase Chromosomes
}

\author{
Christian Zorn*, Thomas Cremer, Christoph Cremer, and Jürgen Zimmer \\ Institut für Humangenetik und Anthropologie der Universität, \\ Albertstr. 11, D-7800 Freiburg i. Br., Federal Republic of Germany
}

\begin{abstract}
Summary. Laser UV microirradiation of Chinese hamster interphase cells combined with caffeine post-treatment produced different patterns of chromosome damage in mitosis following irradiation of a small area of the nucleus that may be classified in three categories: I) intact metaphase figures, II) chromosome damage confined to a small area of the metaphase spread, III) mitotic figures with damage on all chromosomes. Category III might be the consequence of a non-localized distortion of nuclear metabolism. By contrast, category II may reflect localized DNA damage induced by microirradiation, which could not be efficiently repaired due to the effect of caffeine. If this interpretation is right, in metaphase figures of category II chromosome damage should occur only at the irradiation site. The effect might then be used to investigate neighbourhood relationships of individual chromosomes in the interphase nucleus.
\end{abstract}

\section{Introduction}

In many plant and animal species, there is evidence that the chromosomes in the interphase nucleus are arranged in a more or less orderly way (Comings, 1968; Vogel and Schroeder, 1974). Presumably, such an orderly distribution would be functionally important (Barr and Ellison, 1972; Warburton et al., 1973; Vogel and Schroeder, 1974; Singh et al., 1976). However, to discuss such considerations in detail, it is first necessary to know which chromosomes or chromosome segments of a given cell type, at a given stage of the cell cycle, are a) regularly associated with each other, b) regularly not associated, and c) associated at random.

To date there have been three main approaches to these questions (Comings, 1968; Nur, 1973; Vogel and Schroeder, 1974; Singh et al., 1976): (i) observation of the interphase distribution of heterochromatic regions; (ii) statistical evaluation of chromosome arrangement in mitosis; (iii) analysis of chromatid interchanges. Each of these methods has its limitations: (i) does not reveal the distribution of

* To whom offprint requests should be sent 
euchromatic chromosome segments; (ii) is based on the assumption (which has been questioned by Warburton et al., 1973; Schmid et al., 1975; and Singh et al., 1976) that the interphase chromosome arrangement is largely unchanged by the onset of mitosis and the methods of preparation. The validity of (iii) depends on the extent to which the frequency of chromatid interchanges is influenced not only by spatial relationships but by other unknown factors which would give a distorted picture of the internal order of the interphase nucleus (Comings, 1975).

The results of such investigations, particularly for mammalian cells, are contradictory. Numerous papers report a non-random arrangement of metaphase chromosomes (Comings, 1968; Vogel and Schroeder, 1974; Schmid et al., 1975), while other authors find at best only a small tendency towards a non-random distribution (Warburton et al., 1973). In particular, results which concern the association of homologous chromosomes are at variance (Vogel and Schroeder, 1974; Comings, 1975; Hens et al., 1975). Since there is so little agreement on the kinds of spatial relationships that exist between chromosomes in mammalian cells (Warburton et al., 1973), the introduction of new, independent and more direct methods of establishing the internal order of the interphase nucleus seems highly desirable. Such an approach may be offered by UV microirradiation:

Using a laser UV microbeam of wavelength $257 \mathrm{~nm}$ with a minimum spot diameter of $0.5 \mu \mathrm{m}$ (Cremer et al., 1974) it has been possible to apply energy densities of several thousands ergs $/ \mathrm{mm}^{2}$ to small areas of the interphase nucleus of Chinese hamster cells without the cells losing their ability to divide (Cremer et al., 1976). If the damaged chromosomes could be detected during the following metaphase, then it would be possible to deduce which chromosomes were adjacent at the site and time of irradiation. Such an approach applies to both heterochromatin and euchromatin, it is obviously independent of possible effects of microirradiation on the arrangement of chromosomes in subsequent phases of the cell cycle (Sakharov et al., 1976) and of disturbances caused by preparative procedures used to obtain suitably spread metaphase figures.

Here we report a possible method of detecting the damaged chromosome sites in the metaphase following UV microirradiation of the interphase nucleus. In rodent cells, post-replication repair, which would be expected to occur in the irradiated part of the nucleus, can be inhibited by methylated oxypurines such as caffeine (Kihlman, 1974). It is also well known that caffeine potentiates UVinduced chromosome aberrations, and it has been suggested (Kihlman et al., 1974; Nilsson and Lehmann, 1975), that it is the caffeine inhibition of post-replication repair which enhances the production of chromosomal defects. If this suggestion is right, then UV microirradiation of interphase cells and post-treatment with caffeine should result in selective damage to chromosomes lying at the irradiation site.

\section{Material and Methods}

\section{Laser UV Microbeam}

The laser UV microbeam has been decribed in detail elsewhere (Cremer et al., 1974; Cremer et al., 1976). Briefly, a coherent UV beam of wavelength $257 \mathrm{~nm}$, obtained by frequency doubling of an argon ion laser beam of wavelength $514 \mathrm{~nm}$, is focussed into the object plane of the irradiation 
microscope by means of a quartz microscope objective. Here, we used a Zeiss Ultrafluar $32 \mathrm{x} / 0.40$ $\mathrm{Ph}$ Glyz giving a spot with a diameter of approx. $1 \mu \mathrm{m}$. The irradiation objective is used simultaneously for observation of the target cells in phase contrast. Aiming is performed by means of crosshairs in the ocular.

\section{Cell Material}

Diploid fibroblastoid Chinese hamster cells were obtained from lung tissue of a 3-week-old female animal. Cells of passage numbers between 5 and 10 were used for experiments. Some experiments were performed with cells of a subline of V 79 Chinese hamster cells (see Cremer et al., 1976). Cells were grown in Eagle's MEM supplemented with nonessential amino acids and $10 \%$ fetal calf serum (FCS) in a humidified atmosphere with $5 \% \mathrm{CO}_{2}$ at $37^{\circ} \mathrm{C}$.

\section{Irradiation, Post-treatment, and Chromosome Preparation}

For irradiation cells were sown on plastic Petri dishes (Nunc/Denmark, $\varnothing 5 \mathrm{~cm}$ ). One or 2 days later the medium was replaced by MEM without FCS supplemented with HEPES buffer to keep the $\mathrm{pH}$ at 7.4, and the dishes were transferred to the irradiation chamber (Cremer et al., 1976). All cells contained in a square of $2 \times 2 \mathrm{~mm}^{2}$ marked by scalpel cuts in the bottom of the dish were microirradiated in the nucleus. Irradiation was performed at room temperature. Cells which showed no clear interphase nucleus morphology (approx. 15\%) were not irradiated. After microirradiation the cells were incubated in MEM with $10 \% \mathrm{FCS}$ and caffeine at $37^{\circ} \mathrm{C}$ for various times. One $\mu \mathrm{g} / \mathrm{ml}$ colchicine was added $3 \mathrm{~h}$ before in situ chromosome preparation. The cells were exposed to a hypotonic solution ( $15 \%$ aqueous calf serum) for $30 \mathrm{~min}$ at room temperature. Thereafter the hypotonic solution was gradually replaced by fixative (acetic acid/methanol, 1:3), and finally the dishes were air-dried. In control experiments cells were treated with caffeine either without irradiation or with whole-cell irradiation while other cells were microirradiated without caffeine treatment.

\section{Results and Discussion}

A total of 487 diploid Chinese hamster cells were microirradiated in the nucleus, incubated for $26 \mathrm{~h}$ in the presence of $0.5 \mathrm{mM}$ caffeine in the medium, and the chromosomes were prepared in situ. These cells and 73 cells which were not irradiated (no clear interphase morphology; see Material and Methods) yielded 34 metaphase figures which could be divided into three morphological categories; (I) 20 mitoses with no morphological alteration in any chromosome, (II) 7 mitoses with damaged chromosomes in a small part of the metaphase figure with most of the chromosomes outside this area apparently intact (Fig. 1a), (III) 7 mitoses with structural defects in all chromosomes up to complete pulverization (Fig. 1 b and c). Of 78 metaphase figures scored from nonirradiated, caffeine treated control cells, contained in the same Petri dishes as the irradiated ones, 69 were completely intact; only 2 figures showed more than one defect (two chromosome constrictions plus two gaps and one exchange figure plus one fragment, respectively), and these were distributed at different sites on the metaphase plate. Similar results were obtained also with V 79 cells (to be published in detail elsewhere). Figure 1d shows a metaphase found among these cells (post-treatment: $13 \mathrm{~h}$ incubation in the presence of $1 \mathrm{mM}$ caffeine) representing an example of category II.

Intact metaphase figures (category I) might be derived either from cells which were not irradiated for technical reasons (see Material and Methods), or from cells irradiated at the G2 stage of the cell cycle, which seems to be intensitive to the 


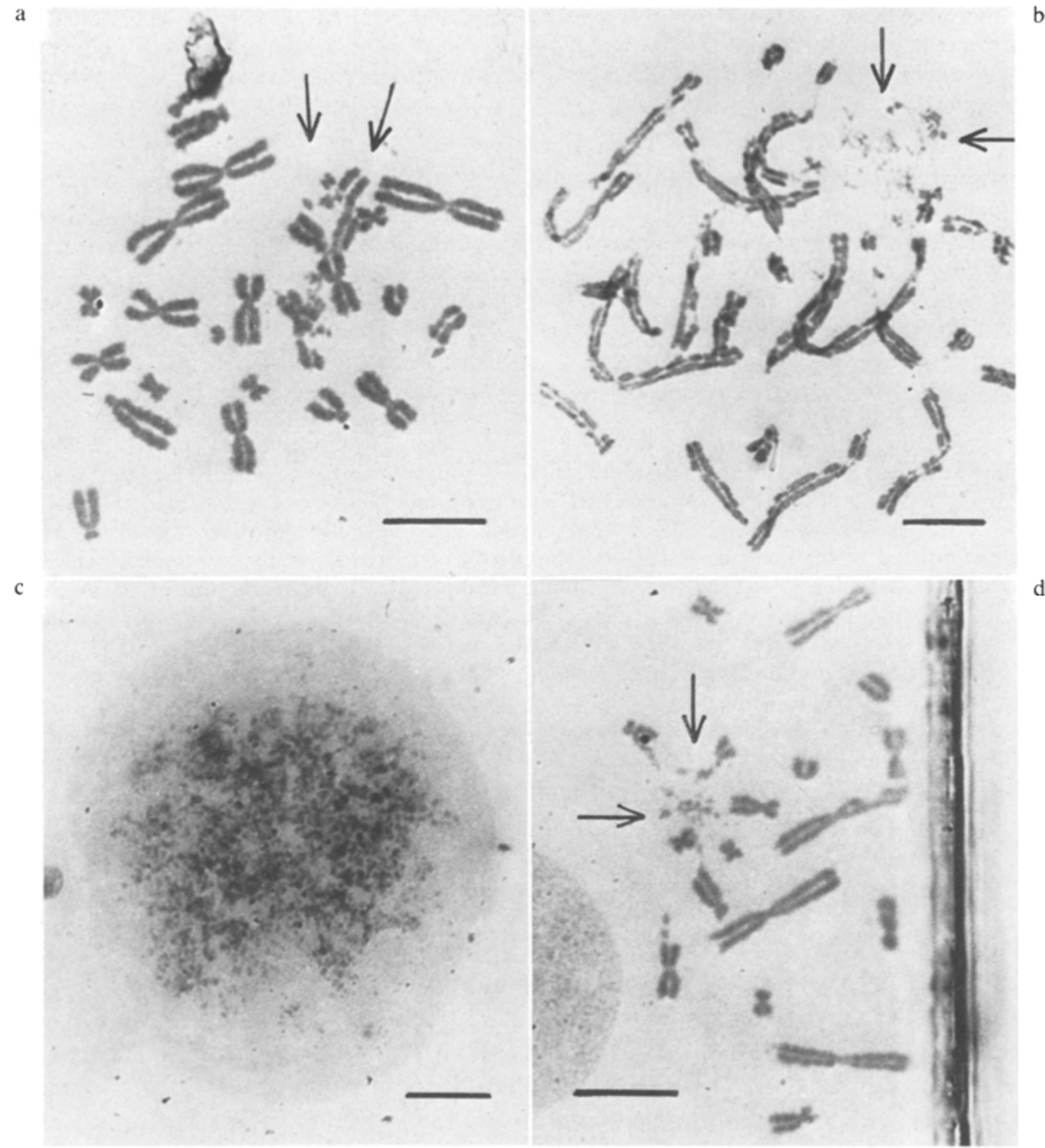

Fig. 1. Mitotic figures showing chromosomal damage obtained after laser UV microirradiation of interphase nuclei of Chinese hamster cells, combined with caffeine post-treatment. (a-c) Mitotic figures of diploid cells; (a) chromosome damage in a limited area (arrows); the rest of the chromosomes are intact (for karyotype see Fig. 2d); (b) pulverization in a limited area (arrows) the rest of the chromosomes showing numerous breaks (endomitotic figure); (c) pulverization of all chromosomes; (d) mitotic figure of a V 79 subline cell showing pulverization in a limited area (arrows). Bars indicate $10 \mu \mathrm{m}$ Staining: Diamond fuchsine

potentiating effect of caffeine (Kihlman, 1974) or finally, from microirradiated cells which succeeded in completing post-replication repair and chromosome condensation despite the presence of caffeine.

Severe local damage (category II, Fig. 1 a and d) was not found in controls or in 25 metaphase figures obtained from cells (V 79) which were microirradiated in the 
nucleus but not treated with caffeine, though the latter included one mitotic cell with complete chromatin pulverization. A total of 505 metaphase figures scored after whole-cell irradiation of interphase cells using a similar wavelength $(254 \mathrm{~nm})$ and caffeine treatment yielded damaged chromosomes distributed over the whole metaphase plate, but no case of local pulverization. Figure 2 shows the karyotypes of four cells with damaged chromosomes concentrated around one "focal point", demonstrating that different chromosomes were damaged in different microirradiation experiments. These metaphase figures are consistent with the hypothesis that inhibition of post-replication repair caused aberrations to appear in microirradiated chromosomes.

This hypothesis is based on the assumption that laser UV microirradiation generates photo products in DNA in only a small part of the nucleus. This assumption is justified by the finding that unscheduled DNA synthesis (excision repair) can be induced selectively at the irradiation site (Moreno and Salet, 1974; Sakharov et al., 1976). In autoradiographs made from euploid human and Chinese hamster fibroblasts after laser UV microirradiation of the nucleus with a focus diameter of $1 \mu \mathrm{m}$, we found ${ }^{3} \mathrm{H}-\mathrm{TdR}$ incorporation in cells not in $\mathrm{S}$ phase. Incorporation is limited to an area of $2-3 \mu \mathrm{m}$ diameter at the focal site (unpublished data). The area of incorporation is larger than the focus because DNA photo products (e.g. thymine dimers, which are expected to occur in a

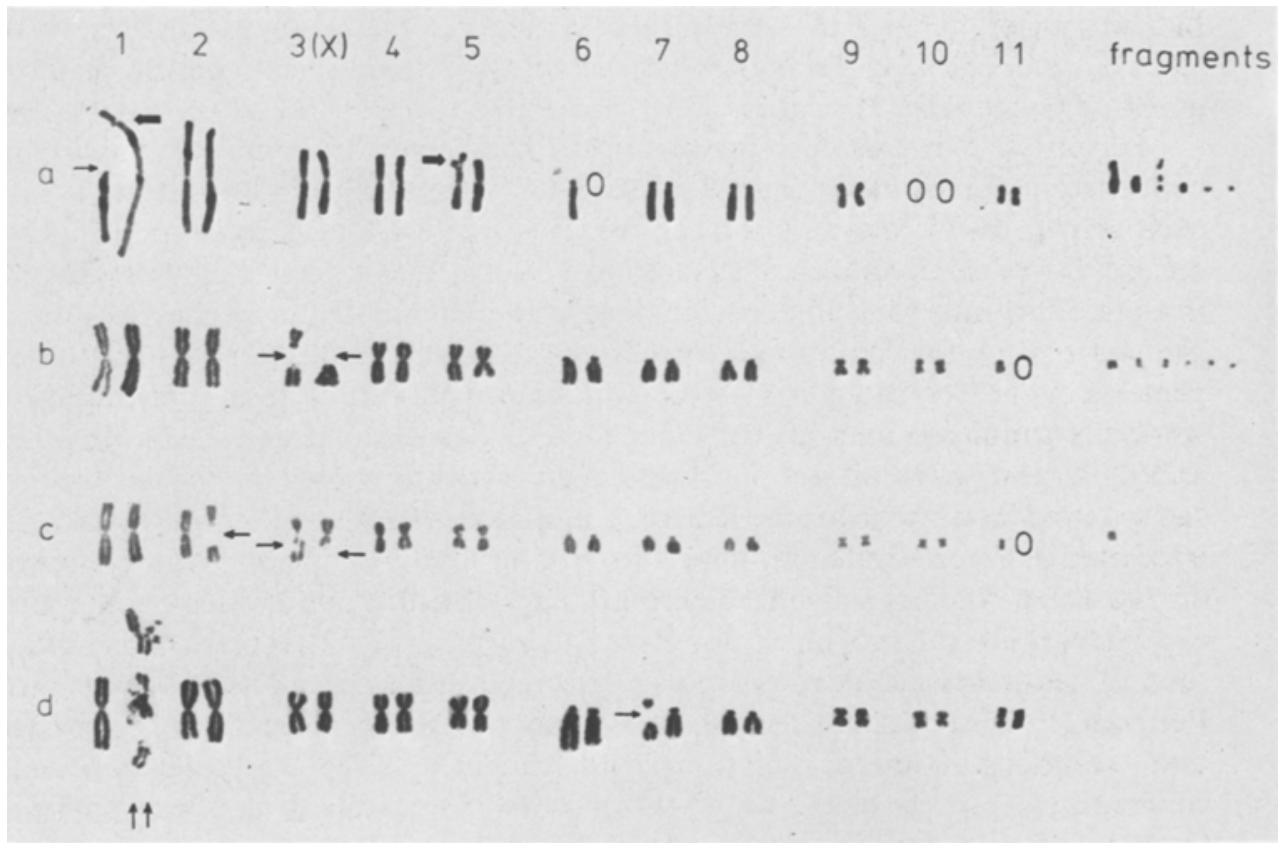

Fig. 2. Four karyotypes (a-d) of diploid Chinese hamster cells after laser UV microirradiation of interphase nuclei and incubation with caffeine. Deletions, chromatid and isochromatid breaks are indicated by light arrows, exchange figures by heavy arrows, "0" indicates a whole chromosome missing or fragmented. Fragments which could not be attributed to one of the chromosomes are shown on the right-hand side 
relatively large quantity at the irradiation site: Cremer et al., 1976) are produced in the whole cone of UV rays within the nucleus. These results indicate that with a laser UV microbeam it is possible to restrict chromatin damage to about onetwentieth to one-thirtieth or even less of the whole nuclear area.

Defects which occurred in all the chromosomes of the metaphase figure (category III, Fig. $1 \mathrm{~b}$ and $\mathrm{c}$ ) indicate another effect of microirradiation combined with caffeine treatment. It is not clear whether overall damage is caused by microirradiation of some special part of the nucleus or nuclear envelope or whether the decisive factor is the stage of the cell cycle at which microirradiation occurs. In the case shown in Figure $1 \mathrm{~b}$ we suggest that the pulverized area represents those chromosomes within the cone of UV rays. Defects in the other chromosomes are presumably not caused by an inhibition of post-replication repair but by a more general effect of caffeine on UV-irradiated cells. Kihlman (1974) suggests that caffeine at high doses may induce metabolic disturbances in chromosomal replication. Microirradiation might itself cause an unspecific disturbance to the metabolism of the whole nucleus and so enhance the sensitivity of all the chromosomes to caffeine. This suggestion is borne out by the fact that the percentage of mitotic cells showing complete chromatin pulverization after microirradiation of the interphase nucleus increases with UV dose (unpublished results obtained with the $\mathrm{V} 79$ subline). An extended discussion of the phenomena obtained in category III will be given elsewhere (in preparation). With respect to the possible use of the method in determining the arrangement of chromosomes in the interphase nucleus it is important to notice that metaphase figures with localized damage (Fig. 1 a and d) can be clearly distinguished from those with overall damage (Fig. $1 \mathrm{~b}$ and $\mathrm{c}$ ).

Pulverized chromosomes in the mitotic cells (Fig. 1) resemble prematurely condensed chromosomes during S phase (S PCC) (Sperling and Rao, 1974). It has been shown that UV irradiation can induce discrete G1 PCC to adopt a highly diffuse S-like morphology (Schor et al., 1975). We suggest that UV microirradiation of interphase nuclei combined with caffeine treatment may lead to a pronounced "interchromosomal asynchrony" (Sperling and Rao, 1974) in a certain percentage of cases (category II) so that the majority of chromosomes enter mitosis while the irradiated ones are still in a more or less decondensed stage (Fig. 1 a and d). Category III mitoses might reflect an asynchrony between the decondensation/condensation sequence (Sperling and Rao, 1974) of all chromosomesirradiated and non irradiated - and the onset of mitosis in the cell. At present we do not know whether pulverized chromosomes obtained in our microirradiation experiments are still in S phase or not. In category II (Fig. 2) a comparison of the sites of chromosomal damage and of late replicating chromatin (Deaven and Petersen, 1973) shows that the two sites do not necessarily coincide. We conclude that the effect of microirradiation is not simply to delay the replication and condensation process of the whole chromosome set equally. If that were so then fragmentation in category II would be confined exclusively to the late replicating, heterochromatic sites as found in the case of S PCC during late S phase (Sperling and Rao, 1974).

It is interesting to note that we find examples where both homologues are simultaneously damaged, while in other cells one chromosome is severely 
damaged, and the homologue remains completely intact. If our line of reasoning is correct then these results indicate that the homologues were somatically associated in the first case but not in the second. More data are necessary to decide whether these are random associations.

Acknowledgements. We thank Prof. U. Wolf and Dr. W. Vogel for discussions and Dr. C. M. Bate for valuable help with the English manuscript. Parts of these investigations will be presented as a doctoral dissertation to be submitted by C. Z. to the Faculty of Biology, Freiburg i. Br. This work was supported by grants of the Deutsche Forschungsgemeinschaft (SFB 46).

\section{References}

Barr, H. J., Ellison, J. R.: Ectopic pairing of chromosome regions containing chemically similar DNA. Chromosoma (Berl.) 39, 53-61 (1972)

Comings, D. E.: The rationale of an ordered arrangement of chromatin in the interphase nucleus. Amer. J. hum. Genet. 20, 440-460 (1968)

Comings, D. E.: Implications of somatic recombination and sister chromatid exchange in Bloom's syndrome and cells treated with mitomycin C. Humangenetik 28, 191-196(1975)

Cremer, C., Cremer, T., Zorn, C., Schoeller, L.: Effects of laser uv-microirradiation $(\lambda=2573 \AA)$ on proliferation of Chinese hamster cells. Radiat. Res. 66, 106-121 (1976)

Cremer, C., Zorn, C., Cremer, T.: An ultraviolet laser microbeam for $257 \mathrm{~nm}$. Microsc. Acta 75, $331-337$ (1974)

Deaven, L. L., Petersen, D. F.: The chromosomes of CHO, an aneuploid Chinese hamster cell line: G-band, C-band, and autoradiographic analyses. Chromosoma (Berl.) 41, 129-144 (1973)

Hens, L., Kirsch-Volders, M., Susanne, C., Galperin-Lemaître, H.: Relative positions of trypsin banded homologous chromosomes in human $(P)$ metaphase figures. Humangenetik 28, 303-311 (1975)

Kihlman, B. A.: Effects of caffeine on the genetic material. Mutat. Res. 26, 53-71 (1974)

Kihlman, B. A., Sturelid, S., Hartley-Asp, B., Nilsson, K.: The enhancement by caffeine of the frequencies of chromosomal aberrations induced in plant and animal cells by chemical and physical agents. Mutat. Res. 26, 105-122 (1974)

Moreno, G., Salet, C.: Unscheduled DNA-synthesis after ultraviolet microirradiation of the cell nucleus. Radiat. Res. 58, 52-59 (1974)

Nilsson, K., Lehmann, A. R.: The effect of methylated oxypurines on the size of newlysynthesized DNA and on the production of chromosome aberrations after uv-irradiation of Chinese hamster cells. Mutat. Res. 30, 255-266 (1975)

Nur, U.: Random arrangement of chromosomes in a radial metaphase configuration. Chromosoma (Berl.) 40, 263-267 (1973)

Sakharov, V. N., Voronkova, L. N., Pyruzyan, L. A., Lomakina, L. Y.: Partial chromosomal labelling induced by ultraviolet microbeam irradiation of mitotic cells. Nature (Lond.) 260, 784-785 (1976)

Schmid, M., Vogel, W., Krone, W.: Attraction between centric heterochromatin of human chromosomes. Cytogenet. Cell Genet. 15, 66-80 (1975)

Schor, S. L., Johnson, R. T., Waldren, C. A.: Changes in the organization of chromosomes during the cell cycle: Response to ultraviolet light. J. Cell Sci. 17, 539-565(1975)

Schwarzacher, H. G.: Chromosomes in mitosis and interphase. Berlin-Heidelberg-New York: Springer 1976

Singh, R. J., Röbbelen, G., Okamoto, M.: Somatic association at interphase studied by Giemsa banding technique. Chromosoma (Berl.) 56, 265-273 (1976)

Sperling, K., Rao, P. N.: The phenomenon of premature chromosome condensation: Its relevance to basic and applied research. Humangenetik 23, 235-258 (1974)

Vogel, F., Schroeder, T. M.: The internal order of the interphase nucleus. Humangenetik 25, 265-297 (1974)

Warburton, D., Naylor, A. F., Warburton, F. E.: Spatial relations of human chromosomes identified by Quinacrine fluorescence at metaphase. Humangenetik 18, 297-306 (1973) 